Cite this: RSC Advances, 2013, 3, 17440

Received 15th July 2013,

Accepted 23rd July 2013

DOI: $10.1039 / c 3 r a 43637 c$

www.rsc.org/advances

\title{
Blinking statistics of colloidal quantum dots at different excitation wavelengths
}

\begin{abstract}
Martijn H. W. Stopel, Jord C. Prangsma, Christian Blum* and Vinod Subramaniam*
In imaging applications the blinking of quantum dots is an ambiguous phenomenon. Although unwanted in conventional imaging, blinking is of significant relevance for super-resolution microscopy. Recent studies report on excitation-wavelength dependent quantum dot blinking, while other studies do not observe this effect. To investigate this disagreement we have systematically studied the effect of the excitation wavelength on blinking of CdSe/ZnS core-shell quantum dots that are commonly used in imaging applications. We recorded single quantum dot intensity traces for 14 different excitation wavelengths and determined for each excitation wavelength the probability to find short- $(<0.5 \mathrm{~s})$ and long- (>0.5 s) lasting bright state periods. This represents a systematic study of a larger set of excitation wavelengths than heretofore reported in the literature. For the quantum dots analyzed, we find minor variations in blinking with excitation wavelength. These variations do not follow a trend and do not correlate with features in the absorbance spectrum of the quantum dots. Our results show that in practice changing the excitation wavelength for CdSe/ZnS quantum dots does not allow optimization of blinking for e.g. super-resolution microscopy and that the effect of blinking as a function of excitation wavelength is not a salient parameter in imaging applications.
\end{abstract}

\section{Introduction}

Blinking or emission intermittency is the sudden switching of an emitter between emitting and non-emitting states. ${ }^{1}$ Since blinking averages out for ensembles of emitters and is thus undetectable, blinking was only discovered with the development of single molecule detection methods. ${ }^{2}$ Blinking has since been found for many emitter classes; ${ }^{3-11}$ interestingly, the underlying mechanism of blinking varies between emitter classes.

The role of blinking in imaging applications is ambiguous. In conventional imaging, blinking is a detrimental effect as it lowers the effective fluorescence quantum efficiency and label concentration, which in turn lengthens acquisition time. In single molecule tracking experiments blinking is unwanted, as it results in gaps in the trajectories when the emitters are in the dark state. However, in single molecule localization based super-resolution microscopy, blinking or state switching of fluorophores is at the heart of imaging structures beyond the diffraction limit, since it allows for the sequential localization of multiple emitters within one diffraction-limited spot. ${ }^{12-16}$

Clearly, controlling blinking is interesting for both superresolution and conventional microscopy, by allowing one to tune the blinking behavior to the desired application. A relatively simple approach to modulating blinking of organic

Nanobiophysics Group, MESA+ Institute for Nanotechnology and MIRA Institute for Biomedical Technology and Technical Medicine, University of Twente, The Netherlands.E-mail: c.blum@utwente.nl; v.subramaniam@utwente.nl fluorophores is by the addition of chemical additives that result in the formation of metastable dark states of the fluorophores. Blinking control of organic dyes has quickly become a much-used tool in super-resolution microscopy. ${ }^{16,17}$

Another class of emitters that is frequently used for imaging applications are so-called quantum dots. Quantum dots are semiconductor nanocrystals exhibiting favorable photophysical properties such as long term photostability, broad absorption range, narrow emission band and size dependent spectral properties. ${ }^{18}$ Quantum dots show complex blinking behavior that is not yet fully understood. Interestingly, recent reports have indicated that the blinking behavior of quantum dots depends on the excitation wavelength, ${ }^{19,20}$ or that quantum dots show an increased probability for a transition to a dark state at specific excitation wavelengths, after continuous illumination with different excitation wavelengths. ${ }^{21}$ It has been suggested that these changes in blinking behavior with excitation wavelength originate from nonemissive trap states that can only be accessed by higher photon excitation energies. ${ }^{19,20}$ Using the excitation wavelengths would on the one hand allow for purely external control over blinking, without need for additives or sample modifications. On the other hand it would have implications for conventional imaging by allowing one to avoid excitation wavelengths that may lead to increased blinking. However, the changes in blinking characteristics with the excitation wavelength are not supported by other reports, where no clear relation between blinking and 


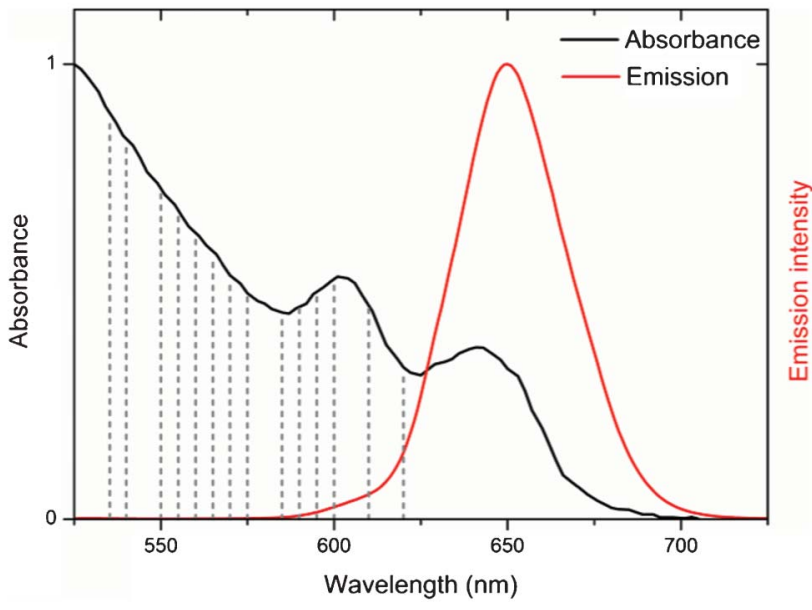

Fig. 1 Normalized fluorescence absorbance and emission spectra of a solution of efluor650 quantum dots. The dashed lines indicate the excitation wavelengths used in this study.

excitation wavelength has been observed for the visible range, while minor effects were observed for an excitation wavelength of $405 \mathrm{~nm}^{22}$

Here we set out to systematically study the blinking of commonly used quantum dots at different excitation wavelengths. We analyzed quantum dot blinking at 14 different fixed excitation wavelengths, ranging from $535 \mathrm{~nm}$ to $620 \mathrm{~nm}$ (Fig. 1). This wide range of excitation covers absorbance regions from the quasi-continuum to close to the band-gap energy of the quantum dots and thus includes excitation energies relative to the band gap that are comparable to earlier studies. For each excitation wavelength we recorded emission time traces from single quantum dots. To discriminate between bright states and weakly emitting states we measure both the emission intensity and average photon arrival time within $10 \mathrm{~ms}$ time bins. To evaluate if the excitation wavelength changes the blinking for time scales relevant for typical imaging applications, we determined for each excitation wavelength the probability to find short-lasting bright periods between 20 milliseconds and $0.5 \mathrm{~s}$ and long-lasting bright periods between $0.5 \mathrm{~s}$ and $3 \mathrm{~s}$. We find minor variations in the blinking of the sampled quantum dots with excitation wavelength. These variations do not show a trend or any features that may be attributed to specific sections of the absorbance spectrum, such as the excitonic peak or the quasicontinuum region. The variations we find are too small to be used effectively to control blinking in imaging. Moreover, our results show that there is no influence of excitation wavelength dependent blinking behavior of quantum dots relevant to conventional imaging applications.

\section{Quantification of blinking}

To study the blinking of single emitters it is necessary to determine accurately when the emitter is in an emitting and when in a non-emitting state. The most common method to determine the times that an emitter resides in a bright, emitting state (sojourn times) is based on an intensity threshold for the identification of states. ${ }^{22-24}$ However, the outcome of the intensity threshold method has been shown to depend significantly on the choice of binning time and threshold level for quantum dots. ${ }^{25}$ One of the reasons is that quantum dot blinking traces exhibit not only a bright, strongly emitting state, but also weakly emitting states and timeaveraged intensity levels between states. ${ }^{26-28}$ To reduce the artifacts that arise due to the thresholding in intensity, a method has been introduced that uses not only the emission intensity, but also the lifetime within a time bin. ${ }^{29,30}$ Here we use this method to identify the bright, strongest emitting state of CdSe/Zns quantum dots, based on the observation that the bright state is characterized by a typical emission lifetime around $20 \mathrm{~ns}$, while the weakly emitting states have a significantly lower lifetime. ${ }^{26-28}$ The average photon arrival time $\left(<t_{\mathrm{a}}>\right)$ reflects differences in decay dynamics between these states. Using both the intensity combined with the average photon arrival time (as an additional discrimination parameter (Fig. 2) $)^{31}$ enhances the contrast between states and provide an additional handle to identify bright states in an intensity-decay plot (ID-plot, Fig. 2b).

To identify time-bins belonging to the bright state and weakly emitting states we plot the intensity versus the average photon arrival time for each time bin (ID plot, see Fig. 2b).

We use a $k$-means cluster analysis ${ }^{32}$ to partition all timebins into two clusters in the ID-plot. This method does not require a prior defined threshold, but iteratively splits the experimentally recorded data set in two clusters, being the onstate and all other weakly emitting states, until these two clusters converge to a maximal statistical separation. Also in this method the discrimination between the clusters follows a curved path in the ID plot, rather than a more limited hard boundary in intensity and average lifetime. The time-bins that
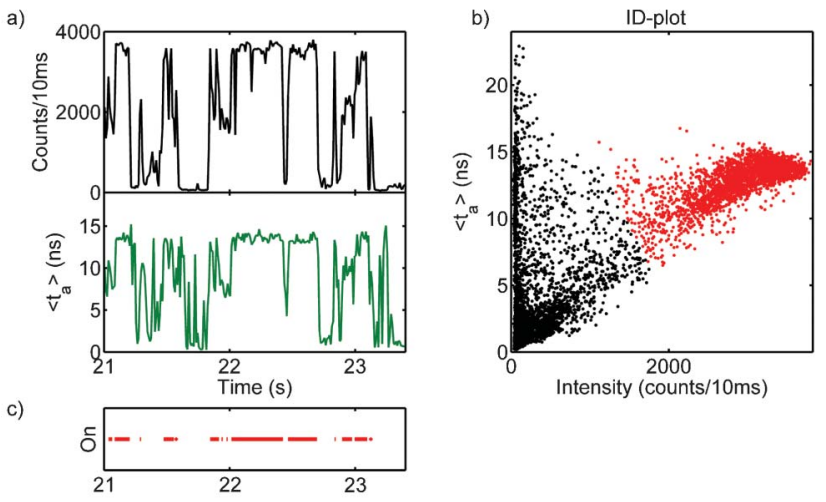

Fig. 2 Cut-out of a time-trace (a) The time-bin size for both the intensity and the average photon arrival time trace is $10 \mathrm{~ms}$. (b) Plotted intensity versus average photon arrival time for all time-bins of the recorded time-trace. Two groups are identified, time bins either belong to the bright state (red) or to weakly emitting states (black). Based on this identification, the sojourn times are determined (see c), bottom panel). 
correspond to the bright state ('on') are marked (Fig. 2b, red points). The sojourn times are calculated from the number of connected time-bins of the bright state using a binary mask (Fig. 2c). These sojourn times are summarized in a probability density histogram which is calculated by the formalism proposed by Kuno et al. ${ }^{33}$

$$
P_{\mathrm{d}}\left(t_{\mathrm{on}}\right)=\frac{N\left(t_{\mathrm{on}}\right)}{\sum N\left(t_{\mathrm{on}}\right)} \cdot \frac{1}{\delta t_{\mathrm{on}}}
$$

where $N\left(t_{\mathrm{on}}\right)$ is the number of events at a defined range $\delta t_{\mathrm{on}}$ around $t_{\mathrm{on}}$. For quantum dots, the probability density histogram typically shows a linear behavior on log-log scale, with a typical slope ranging between -1.2 and $-1.7,{ }^{34-36}$ and slight bending at long sojourn times.

\section{Evaluation of blinking analysis methods}

The probability density histograms generated when using an intensity only based method ${ }^{25}$ have been shown to be very sensitive to the choice of bin time and threshold used. We tested the performance of both the intensity threshold method and the ID discrimination method used here using simulated time-traces of $30 \mathrm{~s}$ lengths. In these simulated traces we assume for simplicity two states, a bright, strongly emitting state of long lifetime, and one weakly emitting state of shorter lifetime. The sojourn times are randomly distributed following a power-law with a coefficient of -1.6 , which we typically find for our quantum dots. The shortest sojourn times that are simulated have a period of $0.1 \mathrm{~ms}$ to allow for state fluctuations that are smaller than the binning time of $10 \mathrm{~ms}$.

As a first test we used an average intensity of 500 counts per $10 \mathrm{~ms}$ and lifetime of $20 \mathrm{~ns}$ for the bright state, and a dim state of average intensity of 100 counts per $10 \mathrm{~ms}$ and $5 \mathrm{~ns}$ lifetime, as observed before. ${ }^{26,27}$ We determined the sojourn times of the simulated dataset using both the intensity threshold and the ID discrimination methods. The statistics of these sojourn times are determined via eqn (1) and the resulting probability density diagrams are shown in Fig. 3a.

As can be expected, we find no clear differences in the performance of both methods when the emission of the two states is clearly distinguished. Both methods show very close resemblance to the theoretically expected probability density curve. We observe bending at long sojourn times in Fig. 3a, that can be assigned to the simulated total time window length of $30 \mathrm{~s}$ per trace, which is the principle upper limit for the sojourn times. Further, the probability density curves show some underestimation at short sojourn times. This is an effect of the high probability to have sojourn times that are shorter than the binning time leading to a high probability to miss state jumps to weakly emitting states. Missed state transitions to weakly emitting states result in an overestimation of long sojourn times. Note that this leads to an underestimation of the steepness of the slope, which is a parameter that is generally used to characterize and quantify blinking statistics.

We then evaluated the performance of both methods for two states that are not clearly separated but have overlapping distributions as it is commonly observed in experimentally recorded single emitter intensity traces. ${ }^{19,23}$ We bring the intensity and decay values of bright and weakly emitting states closer to each other to find a configuration where both methods start to noticeably deviate from the expected result. In Fig. 3b we show the result of our simulation using a bright state intensity of 150 counts per $10 \mathrm{~ms}$ and lifetime of $7.5 \mathrm{~ns}$ and weakly emitting state intensity of 100 counts per $10 \mathrm{~ms}$ and lifetime of $5 \mathrm{~ns}$. Using these parameters both methods show a deviation from the expected result. However, the inclusion of the additional parameter average photon arrival time in the ID method clearly yields a curve that more closely approaches the theoretical values than the intensity threshold method. Note that the plot is on a double logarithmic scale.
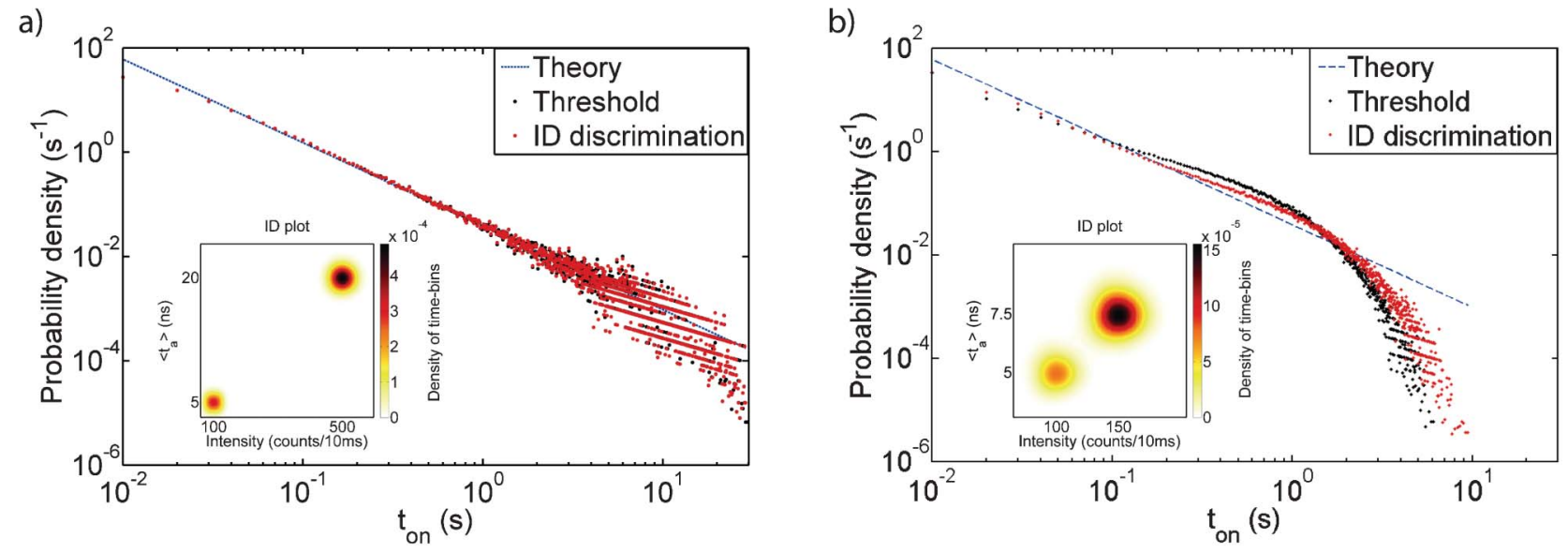

Fig. 3 Probability density histogram of simulated quantum dot blinking traces by a simple two-state blinking model, where the sojourn times are randomly

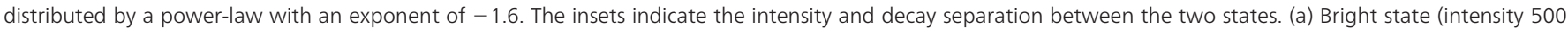
counts per s, lifetime 20 ns); weakly emitting state (intensity 100 counts per s, lifetime 5 ns). (b) Bright state (intensity 150 counts per s, lifetime 7.5 ns); weakly emitting state (intensity 100 counts per s, lifetime $5 \mathrm{~ns}$ ). 
Table 1 The number of analyzed quantum dot time-traces per excitation wavelength

\begin{tabular}{|c|c|c|c|c|c|c|c|c|c|c|c|c|c|c|}
\hline$\lambda_{\text {exc }}(\mathrm{nm})$ & 535 & 540 & 550 & 555 & 560 & 565 & 570 & 575 & 585 & 590 & 595 & 600 & 610 & 620 \\
\hline$N_{\text {dots }}$ & 57 & 37 & 24 & 12 & 636 & 37 & 53 & 57 & 37 & 46 & 49 & 22 & 87 & 35 \\
\hline
\end{tabular}

The deviation of both methods from the expected values arises from the overlap of the statistical distributions of both the intensity and the average photon arrival time of the two states. This effect can be seen in the overlap between the two states in the ID density plot. On the one hand this leads to more short false positives, which shift the probability density curve towards short sojourn times, while on the other hand the decreased intensity contrast leads to an increased underestimation of the probability of short on times.

In our analysis we aim at detecting modifications in blinking behavior by monitoring changes of the probability density histogram. Our simulations show that the shape and bending of the probability curve are insufficiently robust when the state discrimination is not clear. We therefore chose to directly calculate the probability to find short sojourn times and long sojourn times by integrating the area beneath the probability density curve. In this way, the probabilities determined do not depend on any fitting model and are a direct read-out from the data.

\section{Experimental details}

A sample of $1 \mathrm{nM}$ CdSe/ZnS core-shell eFluor650 quantum dots in $2 \%$ wt polyvinylalcohol (PVA) - diluted from a $10 \mu \mathrm{M}$ stock solution - is spin-coated on a clean coverslip at 6000 RPM for one minute. We use a custom built scanning stage confocal microscope based on an Olympus IX71 microscope body in epi-illumination configuration (for details see ref. 37). A PI-527.3CD stage is driven by a PI-E710 digital controller to scan the sample in $x$ and $y$-directions. As excitation source a Fianium SC400 $20 \mathrm{MHz}$ supercontinuum laser combined with an acousto-optical tunable filter (AOTF, Crystal Technologies) to select different excitation wavelengths in the visible range is used. A glass wedge serves as a beam-splitter for all excitation wavelengths and reflects the excitation light through an Olympus 60xW 1.20NA UPlanSApo objective onto the sample. The emission light is collected through the same objective and guided towards an MPD-APD single-photon-counting detector. A Becker\&Hickl SPC-830 TCSPC card measures the arrival times of single photons in respect to excitation pulses. Custom made software was used to control hardware and record data. A Semrock 633SP filter is located at the excitation light path, which constraints the spectral excitation range and suppresses unwanted excitation light. In the detection branch of the microscope a Semrock 633LP and a Semrock 675/67BP filter prevent scattered or reflected excitation light leaking through to the detector and also limit the detection range within the narrow emission band of eFluor650 quantum dots.

\section{Results and discussion}

At 14 different excitation wavelengths, we recorded $30 \mathrm{~s}$ long photon streams for each quantum dot (Table 1). All quantum dots studied were embedded in a film of PVA to avoid any influence of differences in the dielectric environment. ${ }^{38}$ Since it has been shown that blinking statistics depends on excitation power, ${ }^{39,40}$ we adjusted the excitation power to yield equal emission intensity for the different excitation wavelengths. The excitation power that was used was $\sim 1 \mathrm{~kW} \mathrm{~cm}^{-2}$, comparable to the excitation power typically used in other studies. $^{41-45}$

From the recorded photon streams, we calculated intensity and $<t_{\mathrm{a}}>$-traces using a binning time of 10 milliseconds, in agreement with the requirements for objective blinking analyses developed by Crouch et al. ${ }^{25}$ We analyzed all traces that showed a highest intensity cluster above an intensity of 250 counts per $10 \mathrm{~ms}$ and an average photon arrival time above $10 \mathrm{~ns}$ in the ID plots to avoid biasing, e.g. by traces of a weakly emitting state. We quantified blinking as described above using the ID discrimination method. We then constructed one probability density histogram from all sampled quantum dots per excitation wavelengths, according to eqn (1). Because the probability density from a single quantum dot measurement already follows a power law ${ }^{5,33,34}$ we compiled the data from many quantum dots in the blinking analysis to add statistical relevance. ${ }^{2,39}$ In Fig. 4 we present representative probability density diagrams of three excitation wavelengths that range from far above to close to the band-gap energy. Indeed we find

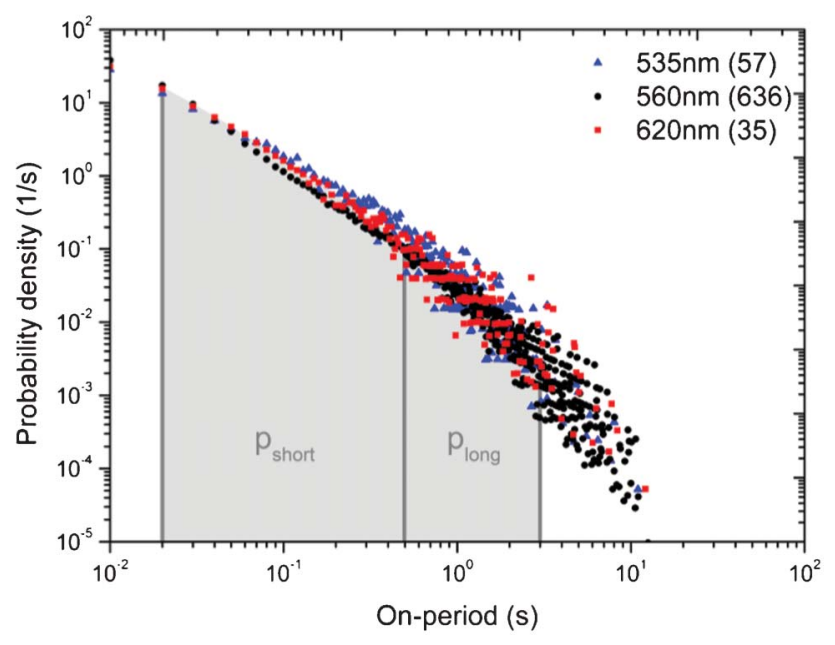

Fig. 4 Probability density diagrams of bright state sojourn times at three different excitation wavelengths ranging from far above to close to the bandgap energy. The numbers in between parentheses indicate the number of quantum dots that are used for the blinking statistics. 
the known clear signature of a power law distribution of the sojourn times for all excitation wavelengths, irrespective of the number of sampled quantum dots per excitation wavelength. The sojourn times range over two decades of time and three decades of probability. For all excitation wavelengths, we find a slope of about -1.6 , which corresponds well with the powerlaw coefficients found in literature ranging between -1.2 and $-1.7 .^{34-36}$ All probability density curves at the 14 different excitation wavelengths are strongly overlapping and show no clear differences.

Changes in the blinking behavior result in changed probabilities to find long or short lasting sojourn times. To quantify the blinking characteristics for different excitation wavelengths we directly determined the probability to find short sojourn times. To meet the Nyquist theorem, we do not consider the $10 \mathrm{~ms}$ sojourn times, which is the time-resolution of our measurements. On the other extreme, the recording window length limits the detection of long sojourn times. Since long sojourn times scarcely occur, we also neglect sojourn times that are longer than $3 \mathrm{~s}$. We directly calculate the probability to find short sojourn times between $20 \mathrm{~ms}$ and $0.5 \mathrm{~s}$ ( $\left.p_{\text {short }}\right)$ and probability to find long sojourn times between $0.5 \mathrm{~s}$ and $3 \mathrm{~s}$ ( $p_{\text {long }}$ ), by integrating the area beneath the probability density curve (Fig. 4). The probability values are obtained from the averaged results of non-parametric statistical bootstrapping ${ }^{46}$ of the probability density curves. The measured set of sojourn times is randomly sampled with replacement until the sample population is equally large as the measured set of sojourn times. We obtained $95 \%$ confidence bounds and average probabilities to find short or long sojourn times from one thousand of these randomly sampled populations. The $95 \%$ confidence bounds indicate the reliability of the probability values that are obtained from the existing population of quantum dots. However, it should be noted that these confidence bounds do not reflect the reproducibility of the parameter values on a different set of quantum dots, meaning that this confidence interval underestimates the true error of the measurement. The result of this analysis is shown in Fig. 5.

We observe that variations in the probabilities to find short $\left(p_{\text {short }}\right)$ and long ( $\left.p_{\text {long }}\right)$ sojourn times for the different excitation wavelengths are between 0.40 to 0.55 and 0.05 to 0.11 , respectively. The probability to find longer sojourn times shows a larger relative variation, which is likely to arise from the fact that the chance to find these long sojourn times is generally low, resulting in a higher uncertainty of the measurement. The observed differences do not follow any recognizable trend, or exhibit any features that may be attributed to specific sections of the absorbance spectrum, such as the excitonic peak or the quasi-continuum region. We observe no prominent changes for excitation wavelengths ranging from far above to close to the band-gap energy.

Our finding that the excitation wavelength has no major effect on quantum dot blinking agrees with earlier results in which no effect of different excitation wavelength in the visible on quantum dot blinking has been observed. ${ }^{22}$ At the blinking

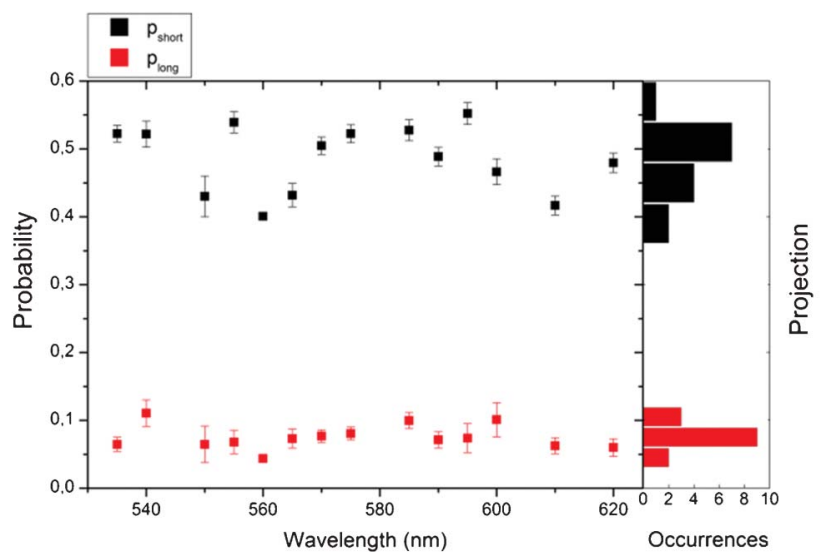

Fig. 5 Probabilities to find sojourn times between $20 \mathrm{~ms}$ and $0.5 \mathrm{~s}$ ( $p_{\text {short, }}$ black) and between $0.5 \mathrm{~s}$ and $3 \mathrm{~s}$ ( $p_{\text {long, }}$ red), for 14 different excitation wavelengths. The probability to find long or short sojourn times varies and shows distributions around $0.49 \pm 0.05$ and $0.075 \pm 0.018$

times up to $3 \mathrm{~s}$ sampled in our study we do not find the distinct differences in quantum dot blinking reported earlier. These earlier studies include one of our own works where we found indications for preferred transitions to a dark state for certain excitation wavelengths after illuminating a single quantum dot with a range of different excitation wavelengths. ${ }^{21}$ However, in this study, as well as in the other studies that report on changed blinking with the excitation wavelengths, ${ }^{19-21}$ the changes have been observed primarily for prolonged illumination and longer timescales outside the range sampled by us in the current study. Due to the power law distribution of the on time duration, on times longer than the $3 \mathrm{~s}$ occur so rarely that influencing this long time blinking has no practical implications on imaging.

\section{Conclusion}

The small variations in blinking that we observe show no trend with excitation wavelengths or any features that may be attributed to specific sections of the absorbance spectrum, such as the excitonic peak or the quasi-continuum region. The small variations observed do not allow for a decided and predictive increase or decrease of blinking. Based on these results, a change of the blinking of quantum dots with excitation wavelength is too small to be used effectively to control blinking in imaging applications and thus does not have to be considered when using quantum dots as luminescent markers.

\section{Acknowledgements}

We gratefully acknowledge the MESA+ Institute for Nanotechnology for funding this research. J. C. P. acknowledges support from STW project nr.12149. 


\section{Notes and references}

1 R. J. Cook and H. J. Kimble, Phys. Rev. Lett., 1985, 54, 1023-1026.

2 T. Sauter, W. Neuhauser, R. Blatt and P. E. Toschek, Phys. Rev. Lett., 1986, 57, 1696-1698.

3 S. A. Patel, M. Cozzuol, J. M. Hales, C. I. Richards, M. Sartin, J. C. Hsiang, T. Vosch, J. W. Perry and R. M. Dickson, J. Phys. Chem. C, 2009, 113, 20264-20270.

4 M. Orrit, Photochem. Photobiol. Sci., 2010, 9, 637-642.

5 F. Cichos, C. von Borczyskowski and M. Orrit, Curr. Opin. Colloid Interface Sci., 2007, 12, 272-284.

6 J. P. Hoogenboom, J. Hernando, E. van Dijk, N. F. van Hulst and M. F. Garcia-Parajo, ChemPhysChem, 2007, 8, 823-833.

7 F. D. Stefani, J. P. Hoogenboom and E. Barkai, Phys. Today, 2009, 62, 34-39.

8 R. Zondervan, F. Kulzer, S. B. Orlinskii and M. Orrit, J. Phys. Chem. A, 2003, 107, 6770-6776.

9 M. Nirmal, B. O. Dabbousi, M. G. Bawendi, J. J. Macklin, J. K. Trautman, T. D. Harris and L. E. Brus, Nature, 1996, 383, 802-804.

10 F. Stracke, C. Blum, S. Becker, K. Mullen and A. J. Meixner, ChemPhysChem, 2005, 6, 1242-1246.

11 C. Blum and V. Subramaniam, Anal. Bioanal. Chem., 2009, 393, 527-541.

12 K. A. Lidke, B. Rieger, T. M. Jovin and R. Heintzmann, Opt. Express, 2005, 13, 7052-7062.

13 T. Ha and P. Tinnefeld, in Annual Review of Physical Chemistry, Vol 63, ed. M. A. Johnson and T. J. Martinez, Annual Reviews, Palo Alto 2012, vol. 63, pp. 595-617.

14 M. Bates, B. Huang, G. T. Dempsey and X. W. Zhuang, Science, 2007, 317, 1749-1753.

15 M. Heilemann, S. van de Linde, M. Schüttpelz, R. Kasper, B. Seefeldt, A. Mukherjee, P. Tinnefeld and M. Sauer, Angew. Chem., Int. Ed., 2008, 47, 6172-6176.

16 J. Vogelsang, C. Steinhauer, C. Forthmann, I. H. Stein, B. Person-Skegro, T. Cordes and P. Tinnefeld, ChemPhysChem, 2010, 11, 2475-2490.

17 M. Heilemann, S. van de Linde, A. Mukherjee and M. Sauer, Angew. Chem., Int. Ed., 2009, 48, 6903-6908.

18 A. P. Alivisatos, Science, 1996, 271, 933-937.

19 K. L. Knappenberger, D. B. Wong, Y. E. Romanyuk and S. R. Leone, Nano Lett., 2007, 7, 3869-3874.

20 K. L. Knappenberger, D. B. Wong, W. Xu, A. M. Schwartzberg, A. Wolcott, J. Z. Zhang and S. R. Leone, ACS Nano, 2008, 2, 2143-2153.

21 C. Blum, F. Schleifenbaum, M. Stopel, S. Peter, M. Sackrow, V. Subramaniam and A. J. Meixner, Beilstein J. Nanotechnol., 2011, 2, 516-524.

22 C. H. Crouch, R. Mohr, T. Emmons, S. Y. Wang and M. Drndic, J. Phys. Chem. C, 2009, 113, 12059-12066.
23 X. Ma, H. Tan, T. Kipp and A. Mews, Proc. SPIE-Int. Soc. Opt. Eng., 2012, SPIE 8232, 82321I-82321I.

24 D. Ratchford, K. Dziatkowski, T. Hartsfield, X. Q. Li, Y. Gao and Z. Y. Tang, J. Appl. Phys., 2011, 109, 103509.

25 C. H. Crouch, O. Sauter, X. H. Wu, R. Purcell, C. Querner, M. Drndic and M. Pelton, Nano Lett., 2010, 10, 1692-1698.

26 G. Schlegel, J. Bohnenberger, I. Potapova and A. Mews, Phys. Rev. Lett., 2002, 88.

27 B. R. Fisher, H. J. Eisler, N. E. Stott and M. G. Bawendi, J. Phys. Chem. B, 2004, 108, 143-148.

28 D. E. Gomez, J. van Embden, P. Mulvaney, M. J. Fernee and H. Rubinsztein-Dunlop, ACS Nano, 2009, 3, 2281-2287.

29 N. Amecke and F. Cichos, J. Lumin., 2011, 131, 375-378.

30 C. Galland, Y. Ghosh, A. Steinbruck, M. Sykora, J. A. Hollingsworth, V. I. Klimov and H. Htoon, Nature, 2011, 479, 203-207.

31 C. Galland, Y. Ghosh, A. Steinbruck, J. A. Hollingsworth, H. Htoon and V. I. Klimov, Nat. Commun., 2012, 3, 908.

32 J. A. Hartigan and M. A. Wong, Journal of the Royal Statistical Society. Series C (Applied Statistics), 1979, 28, 100-108.

33 M. Kuno, D. P. Fromm, H. F. Hamann, A. Gallagher and D. J. Nesbitt, J. Chem. Phys., 2000, 112, 3117-3120.

34 P. Bharadwaj and L. Novotny, Nano Lett., 2011, 11, 2137-2141.

35 P. Frantsuzov, M. Kuno, B. Janko and R. A. Marcus, Nat. Phys., 2008, 4, 519-522.

36 A. V. Malko, Y. S. Park, S. Sampat, C. Galland, J. Vela, Y. F. Chen, J. A. Hollingsworth, V. I. Klimov and H. Htoon, Nano Lett., 2011, 11, 5213-5218.

37 C. Blum, Y. Cesa, M. Escalante and V. Subramaniam, J. $R$. Soc. Interface, 2009, 6, S35-S43.

38 A. Issac, C. Krasselt, F. Cichos and C. von Borczyskowski, ChemPhysChem, 2012, 13, 3223-3230.

39 A. A. Cordones, T. J. Bixby and S. R. Leone, J. Phys. Chem. C, 2011, 115, 6341-6349.

40 K. Goushi, T. Yamada and A. Otomo, J. Phys. Chem. C, 2009, 113, 20161-20168.

41 J. Zhao, G. Nair, B. R. Fisher and M. G. Bawendi, Phys. Rev. Lett., 2010, 104, 157403.

42 R. Schmidt, C. Krasselt and C. von Borczyskowski, Chem. Phys., 2012, 406, 9-14.

43 A. A. Cordones, T. J. Bixby and S. R. Leone, Nano Lett., 2011, 11, 3366-3369.

44 O. Schwartz, R. Tenne, J. M. Levitt, Z. Deutsch, S. Itzhakov and D. Oron, ACS Nano, 2012, 6(10), 8778-8782.

45 B. Fisher, J. M. Caruge, Y. T. Chan, J. Halpert and M. G. Bawendi, Chem. Phys., 2005, 318, 71-81.

46 B. Efron, Biometrika, 1981, 68, 589-599. 